\title{
Convergence Proof of a Class of Adaptive Ant Colony Algorithm
}

\author{
Baojiang Zhao \\ Mudanjing Normal University, Mudanjing Heilongjiang 157011, China \\ baojiangzhao@126.com
}

Keywords: Ant colony optimization, markov process, convergence

\begin{abstract}
This paper presents a class of adaptive ant colony optimization algorithm and proves its convergence properties. The global searching and convergence ability are improved by adaptively changing the pheromone trails evaporation factors and decreasing lower pheromone bound. Markov process analysis is used to prove convergence properties of the algorithms. It is shown that its current solutions of the system converge, with probability one, to an optimal solution of the system.
\end{abstract}

\section{Introduction}

Ant Colony Optimization (ACO) is a class of constructive metaheuristic algorithms that take inspiration from the foraging behavior of real ant colonies [1]. These algorithms have achieved widespread success in solving many different NP-hard combinatorial optimization problems [2, 3]. Still, very little theory is available to explain the reasons underlying ACO's success. Meuleau and Dorigo [4] have shown that ACO algorithms and stochastic gradient descent are strongly related and that a particular form of ACO algorithms converges with probability one to a local optimum. Stützle and Dorigo [5] analyze the MAX-MIN Ant System and show that the algorithm finds at least once an optimal solution with arbitrarily large probability if run long enough. Closely related to the work presented in this paper is Gutjahr's convergence proof [6]. He proved the current solution of a particular ant colony optimization algorithm converges, with a probability that can be made with arbitrarily close to one, to the globally optimal solution. But this limitation has been removed, Gutjahr generalizes the previous result such that the range of application of the convergence result is extended considerably [7]. In another paper [8], Gutjahr analyzes two ACO algorithmic variants which use elitist pheromone update mechanisms and demonstrates that its current solutions converge to an optimal solution with probability exactly one.

From the above analysis, we obtain that algorithmic convergence greatly relies on the change tendency of pheromone trail values. This change can be controlled by pheromone evaporation factor. Moreover, the well-known convergence property of the Simulate Annealing gives us greatly enlightenment. It turns out that a convergence guarantee can be obtained by a suitable speed of “cooling”.

The aim of the present article is to show the theoretical soundness of the ant-based optimization approach. We present a class of adaptive ACO algorithm based on lower pheromone bound, whose evaporation factor and lower pheromone bound are time-dependent. We show that for this particular adaptive ACO algorithm, its current solutions converge, with probability exactly one, to an optimal solution.

\section{The Algorithm}

As it is done in [6], our extension of ACO is also based on a directed graph.

Definition 1. Let an instance of a combinatorial optimization problem be given. By a construction graph for this instance, we understand a directed graph $C=(V, A)$ together with a function $\Phi$ with the following properties:

(1) In $C$, a unique node is marked as the start node.

(2) Let $W$ be the set of (directed) walks $w$ in $C$ satisfying the following conditions:

(i) $w$ starts at the start node of $C$.

(ii) $w$ contains each node of $C$ at most once. 
(iii) The last node on $w$ has no successor node in $C$ that is not already contained in $w$.

Then $\Phi$ maps a subset $\bar{W}$ of the set $W$ onto the set of feasible solutions of the given problem instance. In other words, to each walk $w$ in $\bar{W}$, there corresponds (via $\Phi$ ) a feasible solution, and to each feasible solution, there corresponds (via $\left.\Phi^{-1}\right)$ at least one walk in $\bar{W}$.

Based on Definition 1, the algorithm is the following:

Step 1 Initialize

" ( $k, l)$, set $t(k, l)=1 /|A|$, where $|A|$ is the number of arcs of $C$; set $k$, the current position of the ant, equal to the start node of $C$; set $u$, the current path of the ant, equal to the empty list.

Step 2 Solution Construction

while (a feasible continuation $(k, l)$ of the path $u=\left(u_{0}, \ldots, u_{t-2}, u_{t-1}=k\right)$ of the ant exists) do:

select successor node $l$ with transition probability

$p_{k l}(n, u)=\left\{\begin{array}{cr}\frac{\left.\tau_{k l}(n)\right]\left[\eta_{k l}(u)\right]^{\beta}}{\sum_{r \notin u}\left[\tau_{k r}(n)\right]\left[\eta_{k r}(u)\right]^{\beta}}, & \text { if } l \notin u, \\ 0, & \text { otherwise. }\end{array}\right.$

continue the current path $u$ of the ant to node $l$ by adding arc $(k, l)$ and setting $k:=l$.

Step 3 Pheromone Trail Update

if $(k, l) \in \hat{w}(n)$,then $\tau_{k l}(n+1)=\max \left(\left(1-\rho_{n}\right) \tau_{k l}(n)+\rho_{n} / L(\hat{w}(n)), \tau_{\min }(n)\right)$, otherwise,

$\tau_{k l}(n+1)=\max \left(\left(1-\rho_{n}\right) \tau_{k l}(n), \tau_{\min }(n)\right)$. Where, $\rho_{n}\left(0<\rho_{n}<1\right)$ and $\beta$ are parameters. $\tau_{k l}(n)$ is pheromone values, and $\eta_{k l}(u)$ is desirability values. $\hat{w}(n)$ is the best path found until the end of iteration $n . L(w)$ is the number of arcs of path $w$.

Remark: Because there are only finitely many $\operatorname{arcs}(k, l)$ and only finitely many feasible partial walks $u$, we set

$\gamma=\min \left\{\left[\eta_{k l}(u)\right]^{\beta} \mid(k, l) \in \in w^{*}\right\}>0$, and $\Gamma=\max \left[\eta_{k l}(u)\right]^{\beta}<\infty$. where $w^{*}$ is an optimal walk. Multiplying all desirability values $\eta_{k l}(u)$ by a fixed constant does not change the transition probabilities. So it can be assumed that the values $\eta_{k l}(u)$ are normalized in such a way that $\Gamma=1$, that is, $\left[\eta_{k l}(u)\right]^{\beta}<1$ for all arcs $(k, l)$.

The essential feature of this algorithm is that the evaporation factor $\rho_{n}$ and lower Pheromone bound $\tau_{\min }(n)$ are chosen as time-dependent. .

\section{Convergence}

Before proving the theorem, we introduce three lemmas used in theorem.

Lemma 1. The state variables $X_{n}=(\underline{\tau}(n), \hat{w}(n-1))(n=1,2, \ldots)$ form a Markov process, where $\underline{\tau}(n)$ is the vector of pheromone values $\tau_{k l}(n)$ for all arcs $(k, l)$ at the beginning of iteration $n$ and $\hat{w}(n-1)$ is the best path found at the end of iteration $n-1$.

Lemma 2. Let $\sum_{n=1}^{\infty} \rho_{n}=\infty$, then $\prod_{n=1}^{\infty}\left(1-\rho_{n}\right)=0$.

Lemma 3. Let $\sum_{n=1}^{\infty} \rho_{n}=\infty$, for $B_{n}=\sum_{i=1}^{n}\left[\rho_{i} \prod_{j=i+1}^{n}\left(1-\rho_{j}\right)\right]$, then $\lim _{n \rightarrow \infty} B_{n}=1$.

Theorem 1. Let $\sum_{n=1}^{\infty} \rho_{n}=\infty$ and $\tau_{\min }(n)=\frac{c_{n}}{\log (n+1)}(n \geq 1)$ with $\lim _{n \rightarrow \infty} c_{n}=c>0$. Then, the states $X_{n}=(\underline{\tau}(n)$, $\hat{w}(n-1))$ of the Markov process converge as $n \rightarrow \infty$ to one of the state $\left(\underline{\tau}\left[w^{*}\right], w^{*}\right)$ where $w^{*}$ is an optimal path, and $\underline{\tau}\left[w^{*}\right]$ is defined by $\tau_{k l}\left[w^{*}\right]=\left\{\begin{array}{cc}1 / L\left(w^{*}\right), & \text { if }(k, l) \in w^{*}, \\ 0, & \text { otherwise. }\end{array}\right.$ In particular, there exists with probability one an optimal path $w^{*}$ such that for a fixed ant s, its probability $p_{w^{*}}(n)$ of traversing path $w^{*}$ in iteration $n$ tends to one, as $n \rightarrow \infty$. 
Proof: (1) By $F_{n}$, we denote the event that iteration $n$ is the first iteration in which an optimal path is traversed by some ants. Consider a fixed optimal path $w^{*}$.We have $\neg F_{1} \wedge \neg F_{2} \wedge \ldots \Rightarrow w^{*}$ is never traversed, and hence

$P\left(\neg F_{1} \wedge \neg F_{2} \wedge \ldots\right) \leq P\left(w^{*}\right.$ is never traversed $)=\prod_{n=1}^{\infty} P\left(w^{*}\right.$ not traversed in iteration $n \mid w^{*}$ not traversed in an iteration $\left.i<n\right)$.

Now, for any arc $(k, l)$, it holds that $\tau_{k l}(n) \geq \tau_{\min }(n)=\frac{c_{n}}{\log (n+1)}$. Because of $c=\lim _{n \rightarrow \infty} c_{n}$, there exits $N_{1}$ such that for all $n>N_{1}, \tau_{k l}(n) \geq \frac{c}{2 \log (n+1)}$.

The maximum possible amount of pheromone added to any arc $(k, l)$ after any iteration is $\rho_{n} / L\left(w^{*}\right)$. Hence, due to $\lim _{n \rightarrow \infty} \tau_{\min }(n)=0$, the pheromone trail at sufficiently large iteration $n$ is bounded by

$$
\tau_{k l}(n) \leq\left[\prod_{i=1}^{n}\left(1-\rho_{i}\right)\right] \tau_{k l}(1)+\frac{1}{L\left(w^{*}\right)}\left[\sum_{i=1}^{n}\left[\rho_{i} \prod_{j=i+1}^{n}\left(1-\rho_{j}\right)\right]\right]
$$

According to lemma 2 and lemma 3, this sum converges to $\tau_{\max }=\frac{1}{L\left(w^{*}\right)}$. Hence, there exits $N_{2}$, for all $n>N_{2}$ and all $(k, l) \in A$, such that $\tau_{k l}(n)<1$. Let $|V|$ denotes the maximal numbers of feasible successors of a fixed node. It follows that for $n \geq N=\max \left(N_{1}, N_{2}\right)$ and $(k, l) \in w^{*}$,

$$
p_{k l}(n, u)=\frac{\left[\tau_{k l}(n)\right]\left[\eta_{k l}(u)\right]^{\beta}}{\left(\sum_{r \notin u,(k, r) \in A}\left[\tau_{k r}(n)\right]\left[\eta_{k r}(u)\right]^{\beta}\right)} \geq \frac{\tau_{\min }(n) \gamma}{|V|} \geq \frac{\gamma}{|V|} \frac{c}{2 \log (n+1)}=\frac{c_{1}}{\log (n+1)},
$$

where $c_{1}=\frac{\gamma}{|V|} \frac{c}{2}$. Hence, the probability of a fixed ant $s$ to traverse $w^{*}$ in iteration $n$ is

$$
\prod_{(k, l) \in w^{*}} p_{k l}(n) \geq\left(\frac{c_{1}}{\log (n+1)}\right)^{L\left(w^{*}\right)}(n \geq N) \text {. }
$$

Obviously, this lower bound is independent of what has happened before iteration $n$, such that the estimation also holds conditionally on arbitrary events in iteration $1, \ldots, n-1$. Therefore, an upper bound on the right side of (2) is

$$
\prod_{n=N}^{\infty}\left[1-\left(\frac{c_{1}}{\log (n+1)}\right)^{L\left(w^{*}\right)}\right]
$$

Taking the logarithm of this product, we obtain

$$
\sum_{n=N}^{\infty} \log \left(1-\left(\frac{c_{1}}{\log (n+1)}\right)^{L\left(w^{*}\right)}\right) \leq-\sum_{n=N}^{\infty}\left(\frac{c_{1}}{\log (n+1)}\right)^{L\left(w^{*}\right)}=-\infty
$$

since $\sum_{n}(\log n)^{-L}$ is a diverging series for each positive integer L. As a consequence, the product (6) and also the right side of (2) is zero. Therefore, we obtain $P\left(F_{1} \vee F_{2} \vee \ldots\right)=1$, which proves that the event $F_{1} \vee F_{2} \vee \ldots$ that in some iteration an optimal path is traversed has probability one.

After the iteration where an optimal path has been traversed for the first time, $\hat{w}(n)$ is set equal to this path. Hence, with probability one, the Markov process $X_{n}=(\underline{\tau}(n), \hat{w}(n-1))$ enters in some iteration into one of the sets $\mathrm{R}^{|A|} \times\left\{w^{*}\right\}$, where $w^{*}$ is an optimal path.

(2) We now show that for each $w^{*}$ taken from the set of optimal paths, if the Markov process satisfies $X_{n}(w) \in \mathrm{R}^{|A|} \times\left\{w^{*}\right\}$ for some $n$, then $\lim _{n \rightarrow \infty} X_{n}=\left(\underline{\tau}\left[w^{*}\right], w^{*}\right)$.

Let $m$ denotes the index of the iteration where $w^{*}$ is traversed for the first time, such that the process $X_{n}(w) \in \mathrm{R}^{|A|} \times\left\{w^{*}\right\}$ in iteration $m+1$. Then, in all iterations $n>m$, only $w^{*}$ is reinforced, and $\hat{w}(n)=w^{*}$. By neglecting the possibility of $\tau_{\min }(n)$, it can be easily verified that for $(k, l) \in w^{*}$ and $r=1,2, \ldots$, due to lemma 2 and lemma 3

$$
\tau_{k l}(m+r) \geq\left[\prod_{n=m}^{m+r-1}\left(1-\rho_{n}\right)\right] \tau_{k l}(m)+\frac{1}{L\left(w^{*}\right)}\left[\sum_{i=0}^{r-1}\left[\rho_{m+i} \prod_{j=i+1}^{r-1}\left(1-\rho_{m+j}\right)\right]\right] \rightarrow 1 / L\left(w^{*}\right) \quad(r \rightarrow \infty) .
$$


As a consequence, $\tau_{k l}(m+r)>1 /(2 L)$ for sufficiently large $r$. On the other hand, since $\tau_{\min }(n) \rightarrow 0(n \rightarrow \infty)$, we have $\tau_{\min }(m+r)<1 /(2 L)$ for sufficiently large $r$. Hence, for $(k, l) \in w^{*}$, it holds that $\lim _{r \rightarrow \infty} \tau_{k l}(m+r)=1 / L$.

For $(k, l) \notin w^{*}$, we know $\tau_{k l}(m+r)=\max \left\{\prod_{n=m}^{m+r-1}\left(1-\rho_{n}\right) \tau_{k l}(m), \tau_{\min }(m+r-1)\right\}$.

Because $\prod_{n=m}^{m+r-1}\left(1-\rho_{n}\right) \tau_{k l}(m) \rightarrow 0 \quad(r \rightarrow \infty) \quad$ and $\tau_{\text {min }}(m+r-1) \rightarrow 0 \quad(r \rightarrow \infty)$, we have $\lim _{r \rightarrow \infty} \tau_{k l}(m+r)=0$. Together with $\lim w^{*}=w^{*}$, this yields $\lim _{n \rightarrow \infty} X_{n}=\left(\underline{\tau}\left[w^{*}\right], w^{*}\right)$.

(3) From the above discussion, we know that in some iteration $n$, with probability one, the Markov process $X_{n}(w) \in \mathrm{R}^{|A|} \times\left\{w^{*}\right\}$. It follows immediately from the limits of the pheromone trails and the transition probability that for $(k, l) \in w^{*}$,

$p_{k l}(n, u)=\frac{\left[\tau_{k l}(n)\right]\left[\eta_{k l}(u)\right]^{\beta}}{\left(\left[\tau_{k l}(n)\right]\left[\eta_{k l}(u)\right]^{\beta}+\sum_{(k, r) \notin w^{*}}\left[\tau_{k r}(n)\right]\left[\eta_{k r}(u)\right]^{\beta}\right)} \rightarrow 1(n \rightarrow \infty)$.

So, we obtain $P_{w^{*}}(n)=\prod_{(k, l) \in w^{*}} P_{k l}(n) \rightarrow 1 \quad(n \rightarrow \infty)$.

\section{Conclusion}

It is well known that using constant evaporation factor in pheromone update of the general ant colony algorithm can easily get pheromone value on not reinforced arcs decrease too fast and lead to premature convergence to suboptimal solution. On the other hand, introducing a fixed lower pheromone bound leads to random-search-like behaviour without convergence. As in Simulated Annealing [9], convergence can be obtained by a suitable speed of "cooling" (i.e., reduction of the influence of randomness). Therefore, a compromise method is applied to allow pheromone trails on not reinforced arcs to tend to zero, but slower than constantly. This can be achieved by decreasing evaporation factors and "slowly" lower pheromone bounds.

In this paper, we are not able to give clue to choose the parameter schemes in Theorem to speed up convergence in a best-possible way. It is only presented that if the given conditions are satisfied, the algorithm keeps a suitable balance between the two contradicting aims of exploring the search space and favouring good solution. We could specially emphasize either the first or the second aim, but it is very difficult to determine the optimal point of this range in a theoretical analysis. Meanwhile, we do not affirm that the theoretical convergent speeds indicated by theorem are also the most efficient ones. We only present the convergent conditions of this type ant colony algorithm and get convergence to the optimal solution with probability one.

\section{References}

[1] M. Dorigo, E. Bonabeau and G. Theraulaz: Future Gener. Comput. Syst. vol. 16, p. 851-871(2000).

[2] Christian Blum: Review of "Ant colony optimization" by M.Dorigo, T.Stützle, MIT Press, Cambridge, MA, 2004. Artificial Intelligence . 2005.

[3] Martin Reed, Aliki Yiannakou, Roxanne Evering: Applied Soft Computing Journal. 2014.

[4] N. Meuleau,M. Dorigo: Artif. Life, vol. 8, no.2, p. 103-121, 2002.

[5] T. Stützle, M. Dorigo: IEEE Trans. Evol. Comput. vol. 6, no. 4, p. 358-365, 2002.

[6] W. J. Gutjahr: Probability in the Engineering and Information Science, vol. 17, p. 545-569, 2003.

[7] W. J. Gutjahr: Computers and Operations Research . 2007 (9)

[8] W.J.Gutjahr: Journal of Heuristics, Vol.15,p.227-258,2009.

[9] E. Aarts, J. Korst: Simulated Annealing and Boltzmann Machines, Wiley, 1989. 